\title{
gFORSETI
}

\section{Armonizando tensiones: La necesidad de transparencia en el arbitraje sin destruir la confidencialidad}

\author{
Nicolás Serván Eyzaguirre*
}

Resumen. - En el presente artículo, el autor analiza el paradigma clásico de la confidencialidad en el arbitraje comercial y las posturas de los principales centros arbitrales del mundo. Posteriormente, profundiza sobre la necesidad de transparencia en el arbitraje. Finalmente, plantea una serie de medidas que se deberían poner en práctica para desarrollar la transparencia sin dejar de lado la confidencialidad.

Abstract. - In the present article, the author analyses the classic paradigm of confidentiality in commercial arbitration and the regulations of the main arbitral institutions in the world. Then goes deeper into the need for transparency in arbitration. Finally, he puts forward a series of proposals that should be put into practice to develop transparency without neglecting confidentiality.

Palabras claves. - Arbitraje - Arbitraje Comercial - Confidencialidad - Transparencia.

Keywords. - Arbitration - Commercial Arbitration - Confidentiality - Transparency.

* Estudiante de último año en la Universidad del Pacifico. Practicante en Navarro Sologuren, Paredes y Gray. Ex-presidente de Forseti. Miembro del equipo de Moot de Arbitraje Comercial Internacional de la Universidad del Pacífico. Miembro de ITA Young, ICCA Young y Ciarb Young. 


\section{Introducción}

Un gran sector de expertos en el arbitraje internacional señala que urgen reformas en las que se promueva la transparencia y se deje de lado el paradigma clásico de confidencialidad, que por años se ha asumido como una característica esencial del arbitraje. Parece haberse olvidado las razones por las que la confidencialidad es tan importante para las partes. Un par de ejemplos permitirán centrar nuestra visión.

En un arbitraje que surge de un contrato entre empresas tecnológicas, en donde el aspecto central en controversia radica en la aplicación del know how específico del sector, las partes tienen inmensos incentivos para mantener su controversia en estricto privado. En primer lugar, encontramos que el asunto está relacionado con el secreto tecnológico y comercial, cuyas características en el mercado de la tecnología diferencian de manera clave a las empresas competidoras. Además, el conocimiento sobre cualquier incumplimiento contractual en este sector podría traer graves consecuencias reputacionales para las empresas, situación no deseable desde la perspectiva de las partes.

En casos de esta naturaleza, razonablemente, las partes esperan que la confidencialidad sea inherente al procedimiento arbitral, a fin de que su controversia no salga a la luz con las implicancias que ya hemos descrito.

Por su parte, en un sector en el que los precios son tan determinantes, como en el caso del gas natural, las partes buscan con esfuerzo que los resultados de sus controversias se mantengan en estricta reserva. Las partes buscan no solamente que sus procedimientos de negociación sean confidenciales, sino también cualquier procedimiento arbitral o laudo relacionado a ellos. Por ejemplo, las partes buscan que no sea de conocimiento público el precio de venta en los contratos de aprovisionamiento de gas a largo plazo a clientes libres.

Precisamente, en la guía de Global Arbitration Review sobre arbitrajes de Energía se señaló que

"en el contexto de las controversias sobre el examen de los precios del gas, la confidencialidad que ofrece el proceso de arbitraje es aún más importante, ya que el precio del contrato es una de las informaciones más delicadas de la industria del gas. Por supuesto, la confidencialidad en sí misma no distingue un arbitraje de revisión del precio del gas de un arbitraje comercial. Sin embargo, su efecto se siente de manera bastante diferente. Las cuestiones de confidencialidad suelen plantearse en todas las etapas de las controversias sobre la revisión del precio del gas, incluso durante las negociaciones previas al arbitraje, durante el propio arbitraje y después de que éste haya concluido1".

1 BISHOP, Doak y KAISER, Gordon. The Guide to Energy Arbitrations. Londres: Law Business Research, 2019, p. 214. 
Negar de plano la confidencialidad como característica esencial en el arbitraje comercial, de momento, no aparece como una posibilidad, al menos, desde la perspectiva de los usuarios principales, las partes.

En ese contexto, el presente artículo abordará en primer lugar la idea de confidencialidad en el arbitraje comercial y su tratamiento por los centros arbitrales más importantes en el mundo. Luego, analizaremos la ley de arbitraje peruana y las disposiciones de los principales centros de arbitraje sobre la materia.

Con dicha base, profundizaremos sobre la necesidad imperante de transparencia que se clama desde diversos sectores del arbitraje. Finalmente, realizaremos una serie de propuestas que buscan compatibilizar y armonizar las tensiones existentes y definir líneas claras sobre el alcance de la confidencialidad y la transparencia.

\section{Confidencialidad}

Negar la importancia de la confidencialidad en el arbitraje comercial es cerrar los ojos a lo que buscan las partes cuando asumen los costos de resolver sus controversias. Desde la encuesta de 2010 de Queen Mary University y White E Case, entre aquellos usuarios que tienen una política para su resolución de disputas el $84 \%$ tiene una preferencia por la confidencialidad en el arbitraje2:

\section{Chart 2: Main features of corporations' dispute resolution policies and the extent to which they are mandatory}

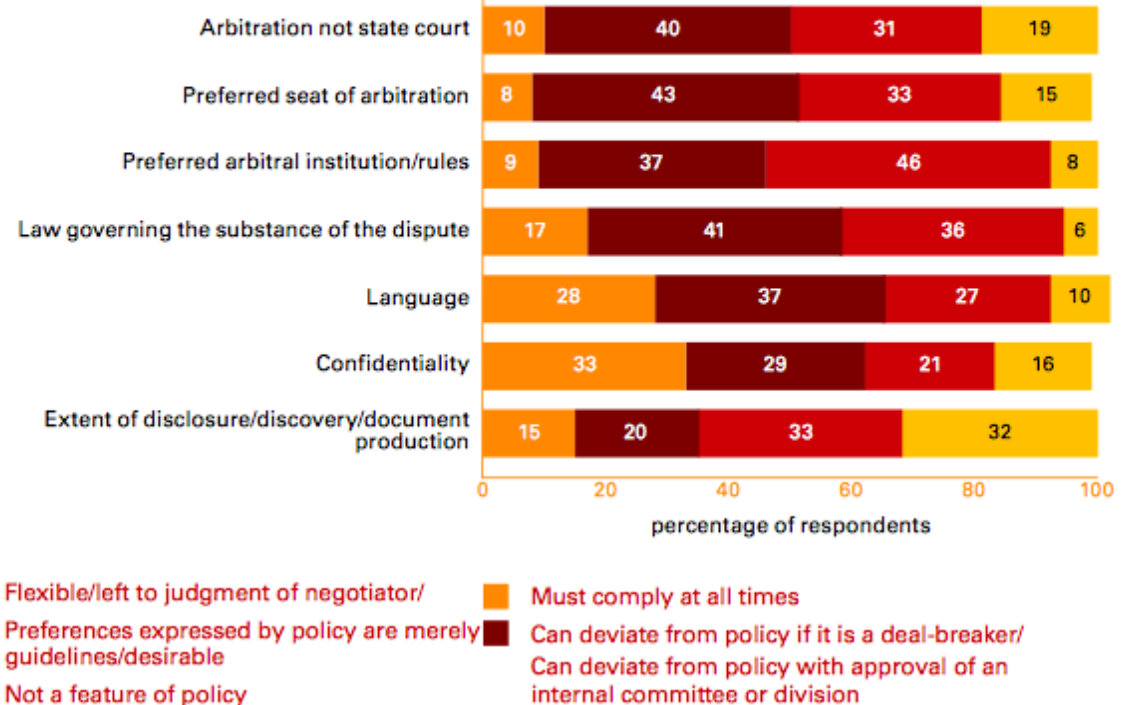

2 White \& Case LLP y Queen Mary University of London. 2010 International Arbitration Survey: Choices in International Arbitration, p. 6. Consulta realizada el día 18 de julio de 2020. Disponible en:

http://www.arbitration.qmul.ac.uk/media/arbitration/docs/2010_InternationalArbitrationSu rveyReport.pdf 
De la misma manera, el $86 \%$ considera importante o muy importante la confidencialidad en el arbitrajes:

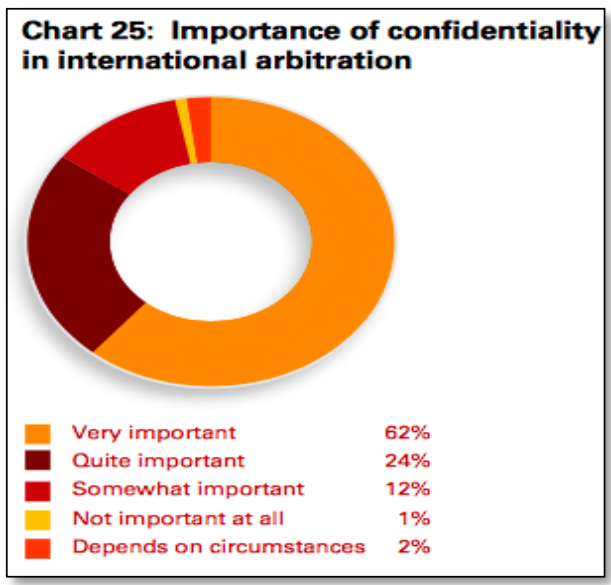

Esta situación también se repitió en la encuesta realizada por ambas instituciones en el año 2015. El 33\% de los encuestados señala que la confidencialidad y privacidad es una de las tres características que más valora del arbitraje internacional4. Inclusive, en la última encuesta realizada por ambas instituciones en el año 2018, el 36\% de usuarios señala que la confidencialidad y la privacidad es una de las características más relevantes5:

$3 \quad$ Ibid, p. 29

4 White \& Case LLP y Queen Mary University of London. 2015 International Arbitration Survey: Improvements and Innovations in International Arbitration, p. 6. Consulta realizada el día 18 de julio de 2020. Disponible en: http:// www.arbitration.qmul.ac.uk/media/arbitration/docs/2015_International_Arbitration_ Survey.pdf

5 White \& Case LLP y Queen Mary University of London. 2018 International Arbitration Survey: The Evolution, p. 7. Consulta realizada el día 18 de julio de 2020. Disponible en: http:// www.arbitration.qmul.ac.uk/media/arbitration/docs/2018-International-ArbitrationSurvey---The-Evolution-of-International-Arbitration 


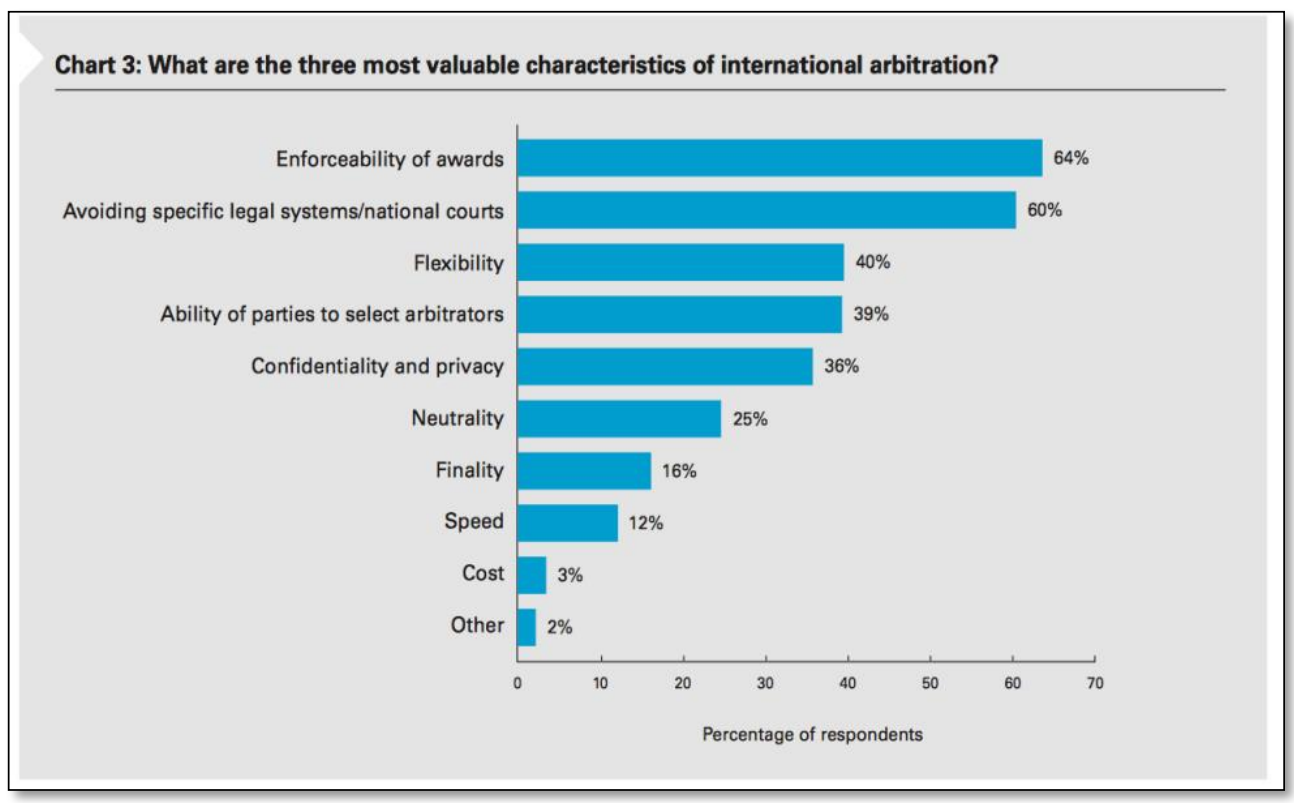

Estos datos no hacen más que afirmar el papel preponderante de la confidencialidad para los usuarios y empresas, pues esta genera una resolución desapasionada y eficaz de conflictos y reduce los riesgos de divulgación de información sensible6. Así, la estadística nos demuestra que "se trata claramente de un concepto que muchos consideran una ventaja fundamental e importante para el arbitraje" 7 . No debemos olvidar que "Inglaterra recibe muchísimos arbitrajes precisamente porque defiende celosamente la confidencialidad 8 ".

Ahora bien, ¿qué debemos entender por confidencialidad y cuáles son sus fronteras? Al respecto, el profesor Born define que "la confidencialidad se utiliza típicamente para referirse a las obligaciones de las partes de no revelar a terceros la información relativa al arbitraje" 9 . Sin embargo, esta también alcanza a los representantes legales y a todos aquellos autorizados a participar en las audiencias10.

No debe confundirse la definición de confidencialidad antes descrita con la privacidad, que también es relevante en el arbitraje. La privacidad está relacionada con el hecho de que las actuaciones arbitrales, principalmente, las audiencias no

6 BORN, Gary. International Commercial Arbitration. Austin: Aspen publishers, 2da Edición, 2014, p. 2782.

7 LEW, Julian, MISTELIS, Loukas A., et al. Comparative International Commercial Arbitration. Kluwer Law International, 2003, p. 8.

8 DE TRAZEGNIES, Fernando. "La publicidad en el arbitraje". En Ius et Veritas, núm. 35, 2007. p. 69.

9 BORN, Gary. International Commercial Arbitration. Austin: Aspen publishers, 2da Edición, 2014, p. 2782.

10 LEW, Julian, MISTELIS, Loukas A., et al. Comparative International Commercial Arbitration. Kluwer Law International, 2003, p. 8. 
están abiertas al público, sino únicamente a las partes, sus representantes y testigosi1.

Durante muchos años, la posición clásica ha sido que la confidencialidad se ha considerado como un elemento natural o implícito del convenio arbitral y del arbitraje. Esto quiere decir que por el solo hecho de existir un convenio arbitral, las partes y los árbitros están obligados por un deber de confidencialidad12. Por ejemplo, en 1998, la Corte de Apelaciones de Inglaterra en el caso Ali Shipping Corporation v Shipyard Trogir señaló que "como cuestión de principio la obligación de confidencialidad (cualesquiera que sean sus límites precisos) surge como corolario esencial de la privacidad de los procedimientos de arbitraje". La Corte propuso el término "que surge de la naturaleza que el propio contrato requiere implícitamente".

Sin embargo, dicha postura ha sido abandonada por muchas jurisdicciones y la confidencialidad no se considera más como un elemento natural del convenio arbitral o del arbitraje. Por ejemplo, Redfern y Hunter citan el caso Esso Australia Resources Ltd $v$ The Honourable Sidney James Plowman and ors, en el que la Alta Corte de Australia señaló que la confidencialidad no es un atributo esencial del arbitraje y que debe ceder ante intereses públicos legítimos frente a autoridades públicas13.

En ese contexto, una de las preguntas esenciales que se desprende de ello es la siguiente: ¿cuáles son los alcances de la confidencialidad? Para responder a ello, es necesario una revisión de las principales leyes de arbitraje y centros de arbitraje del mundo - aquellas sedes e instituciones que atraen más usuarios-, pues no existe uniformidad en la materia14. Sin perjuicio de ello, es preciso tener en mente qué aspectos consideran los usuarios que deben mantenerse confidenciales, en la medida que buscarán sedes o instituciones arbitrales que garanticen la confidencialidad de: el monto de la disputa, los alegatos o escritos, el laudo, los detalles del laudo que permiten la identificación de las partes, las cuestiones jurídicas decididasis.

\section{i. La confidencialidad en la regulación internacional}

11 SMEUREANU, Ileana M. Confidentiality in International Commercial Arbitration. International Arbitration Law Library, Volume 22, Kluwer Law International, 2011, pp. 3-4

12 SILVA ROMERO, Eduardo. "Confidencialidad y transparencia en el arbitraje internacional". En Lima Arbitration, núm. 5, 2012, p. 39.

13 BLACKABY, Niguel, PARTASIDES, Constantine, REDFERN, Alan y HUNTER, Martin. Redfern and Hunter on International Arbitration. Nueva York: Oxford University Press, 6ta edición, 2009, p. 127.

14 TUNG, Sherlin Hsie-Lien \& LIN, Brian. "More Transparency in International Commercial Arbitration: To Have or Not to Have?". En Contemporary Asia Arbitration Journal, 2018, p. 38.

15 White \& Case LLP y Queen Mary University of London. 2010 International Arbitration Survey: Choices in International Arbitration, p. 30. 
En cuanto a las leyes de arbitraje, consideramos pertinente remitirnos a Inglaterra, Estados Unidos, Francia, Singapur y Australia16 por ser algunas de las jurisdicciones arbitrales más importantes.

En lo que respecta a Inglaterra, como ya hemos indicado, en diversos pronunciamientos las Cortes consideraron que la confidencialidad es un elemento natural e implícito del arbitraje17. Sin embargo, los límites de esta obligación no resultaban absolutamente claros $y$ se fueron estableciendo excepciones particulares. Entre las excepciones más importantes encontramos el acuerdo de las partes, una orden judicial o la interposición de un recurso contra el laudo18.

En el caso del Federal Arbitration Act de los Estados Unidos, no existe una disposición expresa con respecto a la existencia de un deber de confidencialidad. En efecto, en 1988 la Corte Distrital de Delaware señaló que no es claro que exista un entendimiento general sobre la confidencialidad en el arbitraje:

"Por ejemplo, la declaración jurada establece que al comienzo del arbitraje de Sonatrach 'se llegó a un entendimiento general por parte del abogado... de que los alegatos y documentos relacionados en el [arbitraje] se mantendrían confidenciales'. (D.I. 76 10, en 5.) Sin embargo, esta afirmación tiene un significado cuestionable. En primer lugar, Begley no señala ningún acuerdo real de confidencialidad, documentado o no. En su lugar, se limita a dar su opinión de que existía un 'entendimiento general'. En segundo lugar, la declaración jurada no indica convenientemente si el entendimiento, si de hecho existió, se alcanzó a petición de Sonatrach o PEPL. Esta distinción es significativa, porque en otra parte de la declaración jurada Begley afirma que 'se dio cuenta de que Sonatrach es extremadamente sensible a las revelaciones de información que considera privada'. (Id. 11, a las 5.) Basado en su experiencia con Sonatrach, Begley 'se formó la opinión' de que la privacidad y la confidencialidad eran esenciales para el éxito de los negocios con Sonatrach. (Id.) A pesar de la opinión de Begley en este sentido, sin embargo, nunca afirma que Sonatrach realmente buscó un entendimiento en cuanto a la confidencialidad $19 "$ ".

Sin embargo, ello no quiere decir que si las partes tienen un acuerdo (ya sea directamente o porque el reglamento arbitral al que se han sometido así lo contempla) con respecto a la confidencialidad no será respetado. Precisamente, la Corte de Apelaciones del Quinto Circuito confirmó una resolución que había declarado procedente una solicitud para ejecutar un acuerdo de confidencialidad contenido en un convenio arbitral 20 .

16 En este caso es relevante dada su particular regulación sobre confidencialidad.

17 Ver por ejemplo, Ali Shipping Corporation v Shipyard Trogir. Además, Born resalta también los casos “Ins. Co. v. Lloyds Syndicate [1995] 1 Lloyd's Rep. 272 (QB) (English High Ct.); Hassneh Ins. Co. of Israel v. Mew [1993] 2 Lloyd's Rep. 243 (QB) (English High Ct.)".

18 YOUNG, Michael y CHAPMAN, Simon. "Confidentiality in International Arbitration: Does the exception prove the rule? Where now for the implied duty of confidentiality under English law?" En ASA Bulletin, vol. 27, núm. 1, 2009, pp. 33 - 36

19 Estados Unidos contra Panhandle Eastern Corp., 118 F.R.D. 346 (1988)

20 ITT Educational Services v. Arce, 533 F.3d 342 (5th Cir. 2008) 
En el caso de Francia, el artículo 1464 del Código de Procedimientos Civiles, que se ocupa de la regulación del arbitraje doméstico establece que "sin perjuicio de las obligaciones legales y salvo estipulación contraria de las partes, el procedimiento arbitral está sometido al principio de confidencialidad". Sin embargo, el artículo 1506 no recoge que esta disposición resulta aplicable al arbitraje internacional, en el cual dependerá del pacto de las partes (directo o indirecto vía un reglamento arbitral).

$\mathrm{Al}$ respecto, los profesores Derains y Kiffer señalan que la concepción de las cortes francesas ha evolucionado "en particular debido a los requisitos de divulgación de las empresas públicas. Los tribunales franceses hacen recaer incluso la carga de demostrar que este principio de confidencialidad existe efectivamente y las razones por las que se plantea sobre la parte que invoca una violación de la confidencialidad 21 ". En este caso, primará el pacto de las partes, pero si no existe acuerdo alguno, se puede recurrir a demostrar el principio de confidencialidad como regla.

En lo que respecta a la confidencialidad en Australia, la ley de arbitraje comercial define la confidencialidad en los siguientes términos:

"Por información confidencial, en relación con las actuaciones arbitrales, se entiende la información relativa a las actuaciones arbitrales o a un laudo dictado en esas actuaciones e incluye lo siguiente

a) El escrito de demanda, la contestación y todos los demás escritos, presentaciones, declaraciones $u$ otra información que una parte proporcione al tribunal arbitral;

b) Toda información suministrada por una parte a otra parte en cumplimiento de una instrucción del tribunal arbitral;

c) Las pruebas (documentales o de otra índole) presentadas al tribunal;

d) Las notas del tribunal arbitral sobre las pruebas orales o los escritos presentados ante el tribunal arbitral;

e) Toda transcripción de las pruebas orales o de los escritos presentados ante el tribunal arbitral;

f) Los fallos del tribunal arbitral;

g) Todo laudo del tribunal arbitral"

A pesar de ello, debemos tener en consideración que existen amplios supuestos regulados en los que se les permite a las partes y a los árbitros revelar información confidencial. Inclusive, se le puede requerir a los árbitros que emitan una orden a través de la cual permitan la revelación de información en supuesto adicionales a los ya regulados. Como hemos señalado con anterioridad, "la confidencialidad no

21 DERAINS, Ives y KIFFER, Laurence. "National Report for France (2013 through 2018)". En Lise Bosman (ed), ICCA International Handbook on Commercial Arbitration, Kluwer Law International, 2018 p. 52. 
es inherente a la aceptación del arbitraje; sin embargo, la Corte [Suprema de Victoria]22 señaló que las partes pueden acordar que los documentos se mantengan confidenciales23".

En el caso de Singapur, los Tribunales han reconocido expresamente la privacidad y confidencialidad, tomando como referencia el modelo inglés24. Las cortes han llegado a afirmar que el principio de justicia abierta no puede dejar de lado la confidencialidad que es reconocida como una consecuencia esencial de la privacidad del arbitraje e implícita al convenio arbitral 25 .

Con respecto a los centros de arbitraje, analizaremos las disposiciones de la Cámara de Comercio Internacional (ICC), las del brazo internacional de la American Arbitration Association (ICDR), las del London Court of International Arbitration (LCIA), las del Singapore International Arbitration Centre (SIAC) y las de Hong Kong International Arbitration Centre (HKIAC).

En el caso de la ICC, prima siempre el acuerdo de las partes. Si no existe un pacto al respecto, se le puede requerir al tribunal que dicte alguna medida relacionada con la confidencialidad del procedimiento o alguna otra materia con el objetivo de proteger información específica26. Si bien no hay duda sobre la competencia del Tribunal, se discute si la parte que solicita la medida debe acreditar el fundamento jurídico de su solicitud, por ejemplo, mediante un acuerdo, la lex arbitri o alguna disposición legal sustantivaz7.

Bajo el reglamento de la ICDR28, existe un deber de confidencialidad que alcanza a los árbitros y a los administradores del arbitraje (el centro), pero deja la

22 En los casos Esso Australia Resources v. Plowman (1995) 183 CLR 10 y Transfield Philippines Inc v. Pacific Hydro Ltd [2006] VSC 175.

23 PRYLES, Michael. "National Report for Australia". En Lise Bosman (ed), ICCA International Handbook on Commercial Arbitration, Supplement No. 104, 2019, p. 34.

24 HWANG, Michael, CHUNG, Katie, et al. "Defining the Indefinable: Practical Problems of Confidentiality in Arbitration". En HKIAC (ed.), International Arbitration: Issues, Perspectives and Practice: Liber Amicorum Neil Kaplan, Kluwer Law International, 2018, p. 74

25 Ibid., pp. 31-32.

26 "Artículo 22. 3) A petición de cualquiera de las partes, el tribunal arbitral podrá dictar órdenes relativas a la confidencialidad de las actuaciones arbitrales o de cualquier otra cuestión relacionada con el arbitraje y podrá adoptar medidas para proteger los secretos comerciales y la información confidencial".

27 DODGE, Kirstin \& SCHRAMM, Dorothee. "Commentary on the ICC Rules, Article 22". En Manuel Arroyo (ed.), Arbitration in Switzerland: The Practitioner's Guide, $2^{\circ}$ edición, Kluwer Law International, 2018 p. 2320

28 "Artículo 37.- Confidencialidad

1. La información confidencial revelada durante el arbitraje por las partes o por los testigos no será divulgada por un árbitro o por el Administrador. Salvo lo dispuesto en el artículo 30, a menos que las partes acuerden lo contrario o que lo exija la legislación aplicable, los miembros del tribunal arbitral y el Administrador mantendrán la confidencialidad de todas las cuestiones relacionadas con el arbitraje o el laudo

2. A menos que las partes acuerden otra cosa, el tribunal podrá dictar órdenes relativas a la confidencialidad del arbitraje o a cualquier asunto relacionado con el arbitraje y podrá adoptar medidas para proteger los secretos comerciales y la información confidencial". 
confidencialidad al acuerdo de las partes o las medidas que dicte tribunal, con el objetivo de proteger secretos comerciales o información confidencial29.

A diferencia de los reglamentos anteriores, el de la LCIA30 va más allá y expresamente prevé el deber de confidencialidad para las partes. Asimismo, este deber alcanza a los árbitros con respecto a las deliberaciones de cualquier decisión. Mientras que, la publicación de laudos está sujeta a acuerdo de las partes y el propio Tribunal. Otro aspecto interesante es que define claramente el alcance del deber de confidencialidad así como las excepciones. A pesar de ello, las partes están facultadas para modificar este estándar de confidencialidad31.

En el reglamento del SIAC32, también encontramos un deber confidencialidad amplio tanto en el aspecto subjetivo como en el objetivo. Las partes, los árbitros y cualquiera que intervenga está obligado por este deber que abarca los procedimientos, los escritos, la evidencia y cualquier material producido en el arbitraje. Además, un aspecto que resalta la importancia de esta obligación es que el Tribunal puede imponer sanciones o condenar a los costos a la parte incumplidora e inclusive las cortes de Singapur ejecutarán los acuerdos sobre confidencialidad 33 .

Finalmente, debemos destacar que el propio reglamento establece las excepciones al a la confidencialidad:

“a. Con el fin de presentar una solicitud a cualquier tribunal competente de cualquier Estado para ejecutar o impugnar el laudo;

29 HOSKING, James \& WALTERS, Gretta. "The ICDR International Arbitration Rules". En Laurence Shore, Tai-Heng Cheng, et al. (eds.), International Arbitration in the United States, Kluwer Law International, 2017, p. 79

30 "Artículo 30.- Confidencialidad

30.1 Las partes se comprometen, como principio general, a mantener la confidencialidad de todos los laudos del arbitraje, así como de todos los materiales del arbitraje creados a efectos del mismo y de todos los demás documentos presentados por la otra parte en el procedimiento que no sean de dominio público, salvo y en la medida en que la divulgación pueda ser exigida a una parte por un deber legal, para proteger o perseguir un derecho legal, o para ejecutar o impugnar un laudo en un procedimiento judicial ante un tribunal estatal $\mathrm{u}$ otra autoridad legal.

30.2 Las deliberaciones del Tribunal Arbitral serán confidenciales para sus miembros, salvo que la legislación aplicable así lo exija y en la medida en que la revelación de la negativa de un árbitro a participar en el arbitraje sea exigida a los demás miembros del Tribunal Arbitral en virtud de los Artículos 10, 12, 26 y 27.

30.3 La LCIA no publica ningún laudo ni ninguna parte del mismo sin el consentimiento previo y por escrito de todas las partes y del Tribunal Arbitral".

31 SCHERER, Maxi, RICHMAN, Lisa, et al. Arbitrating under the 2014 LCIA Rules: A User's Guide. Kluwer Law International, 2015, p. 366

32 "Artículo 39.-Confidencialidad. 39.1 Salvo acuerdo en contrario de las partes, una parte y cualquier árbitro, incluido cualquier árbitro de emergencia, y cualquier persona designada por el Tribunal, incluido cualquier secretario administrativo y cualquier perito, tratarán en todo momento todos los asuntos relacionados con el procedimiento y el laudo como confidenciales. Las discusiones y deliberaciones del Tribunal serán confidenciales".

33 CHOONG, John, MANGAN, Mark, et al. A Guide to the SIAC Arbitration Rules. Oxford University Press, $2^{\circ}$ edición, 2018, p. 30 
b. en cumplimiento de una orden o citación emitida por un tribunal de jurisdicción competente;

c. con el propósito de perseguir o ejecutar un derecho o demanda legal;

d. en cumplimiento de las disposiciones de las leyes de cualquier Estado que sean obligatorias para la parte que hace la divulgación o la solicitud o requerimiento de cualquier organismo regulador $\mathrm{u}$ otra autoridad;

e. en cumplimiento de una orden del Tribunal a petición de una parte con la debida notificación a las otras partes; o

f. a los efectos de cualquier solicitud presentada en virtud de la Regla 7 o la Regla 8 del presente Reglamento".

El reglamento del HKIAC 34 , ante una falta de acuerdo de las partes, estamos ante un amplio deber de confidencialidad que alcanza a las partes, los árbitros, peritos, testigos y la institución arbitral que administra el caso35. Existen determinados supuestos en los que se permite la revelación de información, suficientemente razonables. La publicación de laudos se hará de manera anónima, siempre que no exista objeción alguna.

En todos los casos vistos, siempre primará el acuerdo de las partes. En los reglamentos de LCIA, HKIAC y SIAC existe un deber de confidencialidad expreso que alcanza tanto a las partes como a los árbitros y en el caso del ICDR que solamente alcanza a los árbitros. Los reglamentos de HKIAC y SIAC prevén expresamente sanciones o consecuencias ante el incumplimiento del deber, así como las excepciones al deber de confidencialidad. Por su parte, el reglamento de la ICC no contiene una disposición expresa, sino que señala que, ante la falta de acuerdo entre las partes sobre la materia, se podrá solicitar al Tribunal la adopción de medidas para salvaguardar la confidencialidad de información relevante.

\section{ii. La confidencialidad en la regulación peruana}

En el Perú, la ley de arbitraje se ocupa expresamente de la confidencialidad en el artículo 5136. Al respecto, el profesor Caivano al señalado que: "el artículo 51 ofrece

34 "Artículo 45 - Confidencialidad

45.1 A menos que las partes acuerden otra cosa, ninguna parte o representante de las partes podrá publicar, divulgar o comunicar ninguna información relativa a..:

a) El arbitraje en virtud del acuerdo de arbitraje; o

b) Un laudo o una decisión de emergencia dictada en el arbitraje.

45.2 El artículo 45.1 se aplica también al Tribunal Arbitral, a cualquier árbitro de urgencia, perito, testigo, secretario del Tribunal y al HKIAC".

35 Desde el Reglamento de 2013, HKIAC establece un amplio debe de confidencialidad en el aspecto subjetivo.

HWANG, Michael, CHUNG, Katie, et al. Defining the Indefinable: Practical Problems of Confidentiality in Arbitration. En HKIAC (ed.), International Arbitration: Issues, Perspectives and Practice: Liber Amicorum Neil Kaplan, Kluwer Law International, 2018, pp. 63-64

36 "Artículo 51.- Confidencialidad y publicidad

1. Salvo pacto en contrario, el tribunal arbitral, el secretario, la institución arbitral y, en su caso, los testigos, peritos y cualquier otro que intervenga en las actuaciones arbitrales, están obligados 
una regulación que pretende ser integral sobre el régimen de confidencialidad imperante en el arbitrajesz".

Son pocas las leyes de arbitraje que expresamente se pronuncian sobre la confidencialidad. Como podemos apreciar este deber de confidencialidad es bastante amplio en cuanto al alcance subjetivo pues comprende a las partes, los árbitros, los secretarios arbitrales, la institución arbitral, testigos, peritos y, en general, cualquiera que intervenga en el arbitrajess.

En cuanto al aspecto objetivo, el deber de confidencialidad alcanza a las actuaciones arbitrales, cualquier información que se conozca a través de dichas actuaciones e inclusive al laudo arbitral. Evidentemente, las principales excepciones establecidas son dos: i) la exigencia legal; y, ii) la anulación y/o ejecución del laudo. El profesor Caivano resalta que no se han contemplado supuestos en los que la propia Ley permite la intervención judicial39; sin embargo, consideramos que en ese caso los documentos sometidos al juez dejan de ser confidenciales, pues aplica la excepción para hacer valer un derecho.

Por lo tanto, se trata de un deber bastante amplio, que surge de la ley. Desde nuestra perspectiva, la ley de arbitraje peruana entiende la confidencialidad como un elemento natural del convenio arbitral, en la medida que las actuaciones y toda información ahí revelada es confidencial. Ello, a pesar de que está permitido el pacto en contrario.

En lo que respecta los principales centros arbitrales, analizaremos las disposiciones del reglamento del Centro de Arbitraje de la Cámara de Comercio de Lima (CCL), de la Pontificia Universidad Católica del Perú (PUCP) y de AmCham.

En el caso de la CCL40, la regulación es amplia con respecto al deber de confidencialidad en el aspecto subjetivo y objetivo, siguiente la línea establecida

a guardar confidencialidad sobre el curso de las mismas, incluido el laudo, así como sobre cualquier información que conozcan a través de dichas actuaciones, bajo responsabilidad.

2. Este deber de confidencialidad también alcanza a las partes, sus representantes y asesores legales, salvo cuando por exigencia legal sea necesario hacer público las actuaciones o, en su caso, el laudo para proteger o hacer cumplir un derecho o para interponer el recurso de anulación o ejecutar el laudo en sede judicial (...)".

37 CAIVANO, Roque. "El deber de confidencialidad de los árbitros en el arbitraje comercial desde un enfoque comparativo". En Lima Arbitration, núm. 4, 2010, p 122.

38 MANTILLA - SERRANO, Fernando. "Breves Comentarios a la nueva Ley Peruana de Arbitraje". En Lima Arbitration, núm. 4, 2011, p. 46.

39 "Curiosamente, la ley no contempla otros supuestos en los cuales la misma ley autoriza la judicialización del arbitraje, como son la asistencia que puede requerirse a un juez para la actuación de pruebas, que incluye la posibilidad de que la actuación del medio probatorio se realice ante la autoridad judicial (artículo 45, numerales 1 y 2); o la asistencia para la ejecución forzada de medidas cautelares dispuestas por los árbitros (artículo 48)".

CAIVANO, Roque. "El deber de confidencialidad de los árbitros en el arbitraje comercial desde un enfoque comparativo". En Lima Arbitration, núm. 4, 2010, p. 122.

40 "Artículo 43.-

1. Salvo acuerdo distinto de las partes, estas, sus asesores legales y representantes y, en su caso, los testigos, peritos y cualquier otra persona que interviene en el arbitraje están obligados a mantener la confidencialidad de todos los laudos dictados en el curso del arbitraje así como de las actuaciones arbitrales. Solo se exceptúa su revelación, cuando por exigencia legal, sea 
en la Ley de Arbitraje. Se faculta al centro para la publicación de laudos con fines académicos ocultando el nombre de las partes para evitar su identificación.

Por su parte, el Reglamento de la PUCP regula un amplio deber de confidencialidad 41 , cuyo incumplimiento permite sancionar con multas tanto a las partes como a los propios miembros del Tribunal Arbitral, sin perjuicio de las demás acciones pertinentes. También se han establecido claramente las excepciones aplicables. Finalmente, regula la privacidad de las actuaciones arbitrales.

Finalmente, el reglamento de AmCham regula un amplio deber de confidencialidad que alcanza a los árbitros, secretario, institución y las propias partes con respecto a las actuaciones y cualquier información que pudieran conocer. El incumplimiento de este deber es una falta grave, que inclusive faculta a realizar una inferencia negativa respecto de los intereses de la parte incumplidora por parte del Tribunal y a realizar una condena de costos. Transcurridos 6 meses, se autoriza la publicación del laudo, para fines académicos y/o estadísticos.

Los tres reglamentos antes analizados tienen disposiciones homogéneas. El reglamento de la CCL y el de AmCham se pronuncian sobre la publicación de laudos arbitrales, siempre que se pueda garantizar la confidencialidad de la información de las partes.

Así pues, tomando en consideración la regulación internacional de las principales sedes de arbitraje y las principales instituciones arbitrales del mundo, así como la regulación en el Perú, podemos arribar a tres grandes conclusiones.

En primer lugar, limitadas jurisdicciones consideran que la confidencialidad está implícita en el arbitraje o cuentan con alguna disposición legal expresa42. Sin perjuicio de ello, será el pacto de las partes el que prime en cuanto al deber de confidencialidad, ya sea porque directamente tienen un acuerdo específico sobre

necesario hacer públicas las actuaciones para proteger o hacer cumplir un derecho o cuando tiene por fin ejecutar o recurrir cualquier laudo ante una autoridad judicial competente.

2. El Tribunal Arbitral y los funcionarios y directivos del Centro tienen el deber de mantener la confidencialidad de todas las cuestiones relacionadas con el arbitraje o el laudo.

3. No obstante lo dispuesto en los numerales precedentes, el Centro puede, con fines académicos, publicar laudos o decisiones seleccionadas, de forma íntegra, por extractos o por sumario, siempre que sean previamente editados para ocultar el nombre de las partes y otros datos que permitan su identificación y ninguna parte objete su publicación dentro de los plazos establecidos por la Secretaría para estos propósitos (...)".

41 "Artículo $38^{\circ}$.- Las actuaciones arbitrales son confidenciales. Los árbitros, funcionarios del Centro, los miembros de la Corte de Arbitraje, peritos, las partes, sus representantes legales, sus asesores, abogados, o cualquier persona que haya intervenido en las actuaciones arbitrales, se encuentran obligados a guardar reserva de la información relacionada con el proceso arbitral, incluido el laudo. (...)".

42 SILVA ROMERO, Eduardo. "Confidencialidad y transparencia en el arbitraje internacional". En Lima Arbitration, núm. 5, 2012, p. 42 
la materia o porque se han sometido a las disposiciones de un reglamento arbitral que se pronuncia sobre la confidencialidad.

En cuanto a su alcance, debemos diferenciar el alcance tanto subjetivo como objetivo del deber de confidencialidad. Por lo general, en el aspecto subjetivo, los árbitros, los secretarios arbitrales y el centro tienen un deber de confidencialidad. Bajo ciertos reglamentos, las partes también son alcanzadas por el deber de confidencialidad. En el ámbito objetivo, se incluyen las actuaciones arbitrales y la información producidas en ese contexto, así como el laudo arbitral.

Finalmente, los reglamentos arbitrales que más buscan resguardar la confidencialidad de las actuaciones arbitrales, establecen consecuencias ante el incumplimiento. Así, por ejemplo, facultan a los tribunales arbitrales a imponer sanciones, hacer inferencias negativas o condenar a los costos a la parte que infringe el deber de confidencialidad.

\section{Transparencia}

La transparencia es un principio que se encuentra ampliamente relacionado con el acceso a información, aunque no son lo mismo:

“El acceso público y la transparencia se unen al facilitar el derecho del público a asistir a los procedimientos, así como al permitir el escrutinio de la actuación del juez. Si bien es tentador hacer una amalgama de estos dos conceptos, son sin embargo distintos entre sí en el contexto del arbitraje: el acceso del público es un derecho individual mientras que la transparencia se refiere al sistema en su conjunto. Existe una discrepancia notable en el tratamiento de la transparencia y el acceso público en el arbitraje comercial internacional, dado que el primero se considera a menudo un imperativo mientras que el segundo se considera prescindible. Esta diferencia de trato se deriva de los objetivos que cada concepto trata de alcanzar. Por consiguiente, aunque el acceso público es un instrumento para estimular la transparencia, no es una característica esencial de la transparencia $43^{\prime \prime}$.

Por su parte, Catherine Rogers pone en relieve la diferencia entre transparencia y acceso público, en donde la transparencia busca brindarle información sobre el proceso de decisión a las partes interesadas. Mientras que, el acceso público es un derecho general de los ciudadanos44.

En la encuesta de 2018 de Queen Mary University anteriormente citada se señala que el $28 \%$ de los encuestados considera que uno de los factores que tendrá mayor impacto en el futuro del arbitraje internacional, señala la mayor transparencia45.

43 AVINASH, Poorooye \& FEEHILY, Ronán. Confidentiality and Transparency in International Commercial Arbitration: Finding the Right Balance. Harvard Negotiation Law Review, 2017, p. 283.

44 ROGERS, Catherine. Transparency in International Commercial Arbitration. Penn State Law eLibrary, 2006, p. 1307.

45 White \& Case LLP y Queen Mary University of London. 2018 International Arbitration Survey: The Evolution. P. 7. Consulta realizada el día 18 de julio de 2020. Disponible en: http:// www.arbitration.qmul.ac.uk/media/arbitration/docs/2018-International-ArbitrationSurvey---The-Evolution-of-International-Arbitration 
Ahora bien, la necesidad de transparencia no surge necesariamente de un pedido de los usuarios, sino que busca darle mayor legitimidad al sistema. Así, se ha afirmado que "el arbitraje se considera cada vez más como un sistema opaco para unos pocos que interfiere indebidamente en la capacidad del Estado para proteger los valores, tradiciones e intereses del público46". Ante ello, se señala que

"la transparencia permite al público conocer los casos que implican políticas públicas importantes, como el acceso al agua potable sanitaria, y vigilar esas políticas. Por ejemplo, la divulgación pública de la notificación de arbitraje permite al público conocer la existencia de la controversia y expresar sus opiniones. Aumentando el conocimiento del público de estas políticas, la transparencia puede verse como una expresión de los derechos humanos47".

Sin perjuicio de ello, considero que la necesidad principal de transparencia está basada en la necesidad de generar mayor confianza en la institución como mecanismo de resolución de controversias, pues "el movimiento hacia la transparencia debería conducir a la eliminación de las sospechas, así como a la mejora de los sistemas de resolución de controversias entre inversores y Estados" 48 . Ese movimiento hacia la transparencia o tendencia, ha llevado a que los Estados regulen nuevos estándares de transparencia en los tratados de inversión, a fin tener en cuenta los múltiples intereses involucrados49. Por ejemplo, el TPP regula en su artículo 9.24 expresamente la materia.

Esta tendencia ha llevado a que se realicen diversas medidas tanto en el arbitraje en el que interviene el Estado, el arbitraje de inversiones y el propio arbitraje comercial. Veamos.

\section{i. El arbitraje de inversiones}

La transparencia se ha convertido en parte del día a día en el arbitraje entre inversionistas y Estados. Hay muchas razones para apoyar esa idea. Una de las más importantes es, probablemente, la relevancia o interés público de las decisiones tomadas por los árbitros. La mayoría de los casos involucran asuntos que podrían tener impactos de millones de dólares en los presupuesto públicos e impactos en la regulación. Como explica el profesor Fernández Arroyo:

“En la práctica, es difícil, por una vía o por otra, directa o indirectamente, no considerar que la participación del Estado en un arbitraje de inversiones no terminará afectando intereses generales. En efecto, la sola posibilidad de que el Estado sea condenado a indemnizar a un inversor configura una situación

46 BREKOULAKIS, Stavros. Introduction: The Evolution and Future of International Arbitration. En Stavros Brekoulakis, Julian Lew, et al. (eds.), The Evolution and Future of International Arbitration, International Arbitration Law Library, Kluwer Law International Vol. 37, 2016, p. 3.

47 ZHAO, Mary. Transparency, "International Commercial Arbitration: Adopting a Balanced Approach". En Virginia. Journal of International Law, vol. 59, núm. 2, 2019, pp. 186-187.

48 FERNÁNDEZ ARROYO, Diego. "Current Trends in international commercial arbitration in Latin America". En G. Cordero-Moss (ed.), International Commercial Arbitration. Different Forms and their Features, Cambridge University Press, 2013, p. 401.

49 RUSCALLA, Gabriela. "Transparency in International Arbitration: Any (Concrete) Need to Codify the Standard?" En Groningen Journal of International Law, vol. 3, 2015, p. 22. 
eventual de afectación de intereses generales, toda vez que la suma que el Estado deba afectar a sufragar esa condena implicará una disminución de partidas que podrían ser asignadas a otros fines50".

Los ejemplos de transparencia en el arbitraje de inversiones son bien conocidos. El Centro Internacional de Arreglo de Diferencias Relativas a Inversiones del Grupo del Banco Mundial (CIADI) es la institución más importante en este campo y se enfrentó al reto de adoptar una política de transparencia51. Actualmente, publica información de cada árbitro de su lista de miembros, incluido su currículum vitae y también su participación en otros procedimientos del CIADI, como casos pendientes o concluidos y su participación como expertos.

En un gran número de oportunidades el referido centro publica documentos relevantes de los casos como las demandas o contestaciones y ciertas decisiones (laudos parciales o finales) emitidas por el respectivo Tribunal Arbitral. A raíz de ello, se ha podido crear un amplio repertorio de laudos relevantes de arbitraje inversionista-Estado que son materia de análisis y discusión por la comunidad arbitral52.

Otro elemento que permite destacar claramente la transparencia en el arbitraje inversionista-Estado es la existencia de la Convención de las Naciones Unidas sobre la transparencia en el arbitraje inversionista-Estado. La referida Convención ha sido suscrita por más de 20 países del mundo de diversas regiones y ratificada, hasta el momento, por 5 países53. El objetivo de la convención ha sido facilitar la adopción de la transparencia sin necesidad de modificar los tratados bilaterales de inversión:

“Lo que pretendía hacer la Convención era brindar a todos los Estados que quisieran adoptar la transparencia como criterio general aplicable a todos o a la mayoría de los tratados que reglamentan el arbitraje de inversiones en

50 FERNÁNDEZ ARROYO, Diego. "La transparencia como paradigma en el arbitraje de inversiones". En Attila Tanzi, Alessandra Asteriti, Rodrigo Polanco Lazo y Paolo Turrini (ed). Derecho Internacional de las Inversiones en América Latina, 2016, p. 250.

51 MARTINEZ-FRAGA, Pedro. "Juridical Convergence in International Dispute Resolution: Developing a Substantive Principle of Transparency and Transnational Evidence Gathering". En Loyola University Chicago International Law Review, vol. 10, núm. 1, 2012, p. 52.

52 Para mayor referencia: https://icsid.worldbank.org/en/Pages/cases/AdvancedSearch.aspx

53 A pesar de ello, debemos tener en cuenta que se elaboró bajo un gran consenso y se espera que ese número se eleve. "Tanto el Reglamento como la Convención fueron elaborados por el Grupo de Trabajo II de la CNUDMI sobre Arbitraje y Conciliación (el GT). El GT fue establecido por la Comisión de la CNUDMI para apoyar el mandato de la Comisión de fomentar la armonización y la unificación de la legislación en la esfera del arbitraje y la conciliación. El GT está compuesto por delegaciones de los 60 Estados miembros de la CNUDMI, junto con Estados observadores y observadores de las Naciones Unidas, organizaciones intergubernamentales y no gubernamentales".

SHIRLOW, Esmé. "Dawn of a New Era? The UNCITRAL Rules and UN Convention on Transparency in Treaty-Based Investor-State Arbitration". En ICSID REVIEW - Foreign Investment Law Journal, vol. 31, núm. 3, 2016, p. 624. 
los que ellos participan un instrumento práctico para evitarles el difícil expediente de renegociar cada uno de esos tratados $54^{\prime \prime}$.

Al igual que la confidencialidad tiene límites, la transparencia admite excepciones. Silva Romero admite que se puede proteger información sensible o confidencial como secretos oficiales o comerciales, así como limitar la transparencia cuando esta pudiera afectar el procedimiento arbitral. En este último supuesto pone como ejemplo el caso Biwater Gauff (Tanzania) Limited c. Tanzania, en el que se limitó la transparencia para salvaguardar el procedimiento y que este no se vea expuesto al escrutinio público55. En el mencionado caso si bien se reconoció la tendencia de transparencia en las reglas del CIADI56, consideró necesario hacer un equilibrio entre ello y la integridad del arbitraje y se justifican controles de confidencialidad 57.

\section{ii. El arbitraje con intervención del Estado en el Perú}

Como hemos visto con respecto del arbitraje de inversiones, lo que justifica en gran medida la transparencia es el interés público que subyace en ese foro. Consideramos que una premisa similar se encuentra en el arbitraje con participación de una parte estatal. Desde el Decreto Legislativo 1017, la Ley de Arbitraje establecía la publicidad de los laudos arbitrales58. Sin embargo, mediante el Decreto de Urgencia 020-2020 se ha ampliado esta obligación de publicidad a todas las actuaciones arbitrales, de acuerdo el numeral 3 del artículo 51 de la Ley de Arbitraje:

“3. En los arbitrajes en los que interviene como parte el Estado peruano, las actuaciones arbitrales y el laudo son públicos una vez que ha concluido el proceso arbitral, observando las excepciones establecidas en las normas de transparencia y acceso a la información pública. Cada institución arbitral reglamenta las disposiciones pertinentes. En los arbitrajes ad hoc asume dicha obligación la entidad estatal que intervino en el arbitraje como parte"

Sobre esta modificación se ha señalado que es "coherente, además, con la tendencia internacional de dotar de mayor transparencia al arbitraje. Este último es un aspecto que se justifica aún más en los casos en que el Estado es parte, debido al interés público que hay detrás59".

54 FERNÁNDEZ ARROYO, Diego. "La transparencia como paradigma en el arbitraje de inversiones". En Attila Tanzi, Alessandra Asteriti, Rodrigo Polanco Lazo y Paolo Turrini (ed). Derecho Internacional de las Inversiones en América Latina, 2016, p. 269.

55 SILVA ROMERO, Eduardo. "Confidencialidad y transparencia en el arbitraje internacional". En Lima Arbitration, núm. 5, 2012, p. 51.

56 El Tribunal expresamente señaló que: “Considerations of confidentiality and privacy have not played the same role in the field of investment arbitration, as they have in international commercial arbitration. Without doubt, there is now a marked tendency towards transparency in treaty arbitration".

57 BLACKABY, Niguel, PARTASIDES, Constantine, REDFERN, Alan \& HUNTER, Martin. Redfern and Hunter on International Arbitration. Nueva York: Oxford University Press, 6ta edición, 2009, p. 132.

58 MANTILLA - SERRANO, Fernando. "Breves Comentarios a la nueva Ley Peruana de Arbitraje". En Lima Arbitration, núm. 4, 2011, p. 46.

59 HORNA, Emily. "Principales modificaciones a la Ley de Arbitraje: Decreto de Urgencia No 0202020". Consulta realizada el día 18 de julio de 2020. Disponible en: 
La razón por la que se debe publicitar los laudos en los que interviene el Estado ha sido claramente definida en la exposición de motivos, ya que "se pueden haber resuelto materias que importan al interés general o al uso o afectación de los fondos públicos, situaciones en las que por transparencia, los ciudadanos y autoridades deben contar con acceso a la información pertinente60".

Sobre la publicación de laudos, la Municipalidad Metropolitana de Lima (MML) presentó un recurso de anulación en el que alegó la vulneración del deber de confidencialidad por parte del Tribunal Arbitral. En el transcurso del arbitraje, el demandante presentó como prueba el laudo arbitral emitido en el caso entre Perú Masivo S.A.C. contra la MML. Dicho laudo fue utilizado por el Tribunal Arbitral como fundamento al momento de resolver. La Sala Comercial consideró que no se había vulnerado el deber de confidencialidad en los siguientes términos:

“Teniendo en cuenta lo establecido por la ley que norma el Arbitraje, el Tribunal Arbitral no incurrió en causal de nulidad alguna por contravención al principio de confidencialidad, cuando al emitir pronunciamiento sobre la responsabilidad de la MML (en los fundamentos 186 en adelante del laudo) tomó de base lo resuelto con motivo del Caso Perú Masivo S.A.C. y la Municipalidad Metropolitana de Lima, de fecha 18 de febrero de 2015, ya que se trató de un caso que adquirió la calidad de público por haber terminado sus actuaciones con anterioridad al presente laudo61".

Un aspecto interesante que se desprende de la publicidad de laudos arbitrales es si resulta posible considerar la existencia de una especie de "jurisprudencia arbitral", aunque el término no sea el más adecuado. Consideramos que dada la publicidad de los laudos arbitrales en donde interviene el Estado, resulta factible que sean presentados como sustento de la posición de una de las partes. Sin embargo, no podrán ser de ninguna manera considerados vinculantes para el Tribunal, ya que solamente es vinculante para las partes en controversia62.

Nada impide que el Tribunal utilice los criterios expuestos en otro laudo arbitral63. Sin embargo, no resulta posible imponer como cosa juzgada un laudo a un tercero

https:/ /ius360.com/columnas/principales-modificaciones-a-la-ley-de-arbitraje-decreto-deurgencia-no-020-2020/

60 La Exposición de Motivos de la Ley de Arbitraje de puede encontrar en el Sistema Peruano de Información Jurídica (SPIJ).

61 Expediente 441-2018-01817-SP-CO-02.

62 Una excepción a dicha afirmación se produce cuando se trata de un caso entre las mismas partes, que tiene calidad de cosa juzgada entre ellas y sí resulta vinculante.

63 "El carácter persuasivo de los laudos arbitrales anteriores implica en cierta medida que los árbitros internacionales se ven a sí mismos como parte de un grupo de árbitros internacionales cuya función y razón de ser es satisfacer las necesidades particulares de la comunidad empresarial internacional (...) Si se acepta la idea de ese sistema autónomo de justicia internacional, es perfectamente comprensible que los árbitros internacionales traten de ser lo más coherentes posible".

MOURRE, Alexis \& VAGENHEIM, A. Arbitral Jurisprudence in International Commercial Arbitration: The Case for a Systematic Publication of Arbitral Awards in 10 Questions. Consulta realizada el día 18 de julio de 2020. Disponible en: http:/ / arbitrationblog.kluwerarbitration.com/2009/05/28/arbitral-jurisprudence-in- 
que no formó parte del arbitraje64. En ese caso, debemos recordar que procede presentar un amparo contra un laudo "cuando (...) sea interpuesto por un tercero que no forma parte del convenio arbitral y se sustente en la afectación directa y manifiesta de sus derechos constitucionales a consecuencia del laudo pronunciado en dicho arbitraje65". Por lo tanto, el Tribunal deberá ser cuidadoso y no asumir, por ejemplo, los hechos de otro caso como una verdad vinculante para otra controversia.

\section{iii. El arbitraje comercial}

La gran pregunta que surge de ello es si lo visto con respecto del arbitraje de inversiones y el arbitraje con intervención del Estado se puede replicar, sin más, en el arbitraje comercial. Desde mi perspectiva, la respuesta es negativa.

Como hemos visto, una de las razones por las que se prefiere el arbitraje comercial es por su privacidad y confidencialidad, pues en el arbitraje es inminentemente privado, a diferencia de los intereses públicos relacionados con los arbitrajes de inversión y aquellos en los que participa el Estado. El carácter se debe a la naturaleza contractual del arbitraje como mecanismo de resolución de disputas66. Además, debemos resaltar los árbitros están fuera de cualquier presión pública que pudiera existir en torno al caso, como la presión mediática67. Por lo tanto, la transparencia absoluta podría poner en riesgo el arbitraje.

Ello de ninguna manera implica que la transparencia debe ser nula. No olvidemos que la falta de transparencia causa que las partes desconfíen en las instituciones arbitrales68. Asimismo, "permitiría a los círculos arbitrales evaluar el profesionalismo y la competencia de los (...) Además, dado el secreto inherente al arbitraje, los tribunales no deben permitir que la confidencialidad asfixie la capacidad de exponer cualquier actividad ilícita que pueda haber ocurrido durante las actuaciones arbitrales69". Desde mi perspectiva, a pesar del amplio deber de confidencialidad, considero que no se requiere modificar la ley de arbitraje, a fin de implementar las medidas de transparencia. En primer lugar, porque el artículo 51 permite pacto en contrario, ya sea directa o indirectamente (a

international-commercial-arbitration-the-case-for-a-systematic-publication-of-arbitral-awardsin-10-questions/?doing_wp_cron=1596005411.7288129329681396484375

64 Por ejemplo, A no puede imponerle como cosa juzgada a B un laudo arbitral derivado de un arbitraje entre A y C (sin intervención de B) que reconoce un monto dinerario a favor de $\mathrm{C}$. En ese caso, un nuevo tribunal arbitral deberá analizar nuevamente el caso con efectos entre A y B y no podrá asumir como vinculantes los hechos del caso entre A y C.

65 Caso María Julia. Expediente 00142-2011-PA/TC.

66 BULLARD, Alfredo. "El Dilema del Huevo y la Gallina: El Carácter Contractual del Recurso de Anulación". En Derecho y Sociedad, núm. 38, 2012, p. 18.

67 SILVA ROMERO, Eduardo. "Confidencialidad y transparencia en el arbitraje internacional". En Lima Arbitration, núm. 5, 2012, p. 38.

68 TUNG, Sherlin Hsie-Lien \& LIN, Brian. "More Transparency in International Commercial Arbitration: To Have or Not to Have?" En Contemporary Asia Arbitration Journal, 2018, p. 38.

69 AVINASH, Poorooye \& FEEHILY, Ronán. "Confidentiality and Transparency in International Commercial Arbitration: Finding the Right Balance". En Harvard Negotiation Law Review, vol. 22, 2017, p. 283. 
través de una institución arbitral). Al respecto, Rogers resalta la importancia de los centros arbitrales en la materia:

"Las instituciones de arbitraje han dirigido o promovido muchas de las principales innovaciones en materia de transparencia, lo que no es sorprendente puesto que son los principales responsables de las políticas del sistema de arbitraje comercial internacional. Incluso en los aspectos más sencillos de sus reglamentos de arbitraje, es evidente que, colectivamente, las instituciones arbitrales han ido añadiendo precisión y detalle para atraer a más partes comerciales al sistema y ganar cuota de mercado70".

De hecho, ello se corrobora con las expectativas de los usuarios con respecto a las actuaciones de las instituciones arbitrales Los centros arbitrales tienen clara esta situación, pues reconocen que

"para ser percibidas como legítimas, justas desde el punto de vista del procedimiento y dignas de confianza, las instituciones de arbitraje deben exhibir el máximo nivel de transparencia permitido por sus normas. Los procesos de la institución deben ser, y ser percibidos como tales, disciplinados y basados en reglas, y reflejar una ética de independencia, responsabilidad y profesionalidad71".

\section{Medidas de transparencia en el arbitraje comercial}

Así, haremos un breve repaso sobre las principales medidas que han adoptado los centros de arbitraje más importantes del mundo con respecto a la transparencia y de qué manera pueden replicarse en nuestro país.

\section{i. Publicación de información relevante}

Una importante apuesta de la ICC es la publicación de información relevante de los casos. Así, de acuerdo con las Notas a las partes y al Tribunal Arbitral para la conducción del arbitraje72, para los arbitrajes registrados a partir del 1 de enero de 2016, se publicará la siguiente información: i) los nombres de los árbitros; ii) su nacionalidad; iii) su función en el tribunal; iv) el método de su nombramiento; $y$, v) si el arbitraje está pendiente o concluido. Para los arbitrajes registrados a partir del 1 de julio de 2019, también se publicará el sector de la industria involucrada y los abogados que representan a las partes en el caso.

Además, los centros de arbitraje cuentan con un gran flujo de información que es relevante para las partes. Por ejemplo, uno de los beneficios que se resalta del

70 ROGERS, Catherine. Transparency in International Commercial Arbitration. Penn State Law eLibrary, 2006, p. 1307.

71 Survey: Arbitral Institutions Can Do More to Foster Legitimacy. True or False? En Albert Jan Van den Berg (ed.), Legitimacy: Myths, Realities, Challenges, ICCA Congress Series, Volume 18, Kluwer Law International, 2015, p. 727

72 Consulta realizada el día 18 de julio de 2020. Disponible en: https://cms.iccwbo.org/content/uploads/sites/3/2017/03/ICC-Note-to-Parties-and-ArbitralTribunals-on-the-Conduct-of-Arbitration_spanish.pdf 
arbitraje es su rapidez frente a los procesos judiciales (al menos en países con gran sobrecarga como el Perú). Así, tanto el ICDR73 como SIAC y HKIAC74 publican el tiempo promedio.

Aparte de esa información, tanto LCIA, ICDR, SIAC, HKIAC y ICC publican un reporte con sus estadísticas anuales, que incluyen los montos en disputa, los sectores relacionados con la disputa, la nacionalidad de las partes, la ley aplicable, entre otra información relevante. Ello no solamente genera mayor confianza en estas instituciones, sino que permite a los usuarios tomar mejores decisiones.

En el Perú, la Cámara de Comercio de Lima publica información relevante sobre los arbitrajes que administra a través de su portal del Faro de Transparencia; sin embargo, hasta la fecha no ha publicado estadísticas sobre sus casos. Por su parte, la PUCP ha publicado el número de casos comerciales y casos con intervención de una parte estatal, pero no ha profundizado más en la información. En el caso de Amcham, no se ha encontrado estadística.

Considero que si bien existe se está trabajando en fortalecer la transparencia, esta información no es suficiente. Se requieren mayores estadísticas. Los costos de implementar estas medidas no son altos, pues se trata de sistematizar la información y publicarla.

En cuanto a la información sobre los tribunales, considero esencial esta información, pues los usuarios del arbitraje tendrán mayor información para tomar decisiones, así como para verificar potenciales conflictos de interés. La CCL ha tomado la iniciativa, por lo que, no vemos mayores inconvenientes en que los otros centros implementen reformas en la misma línea.

\section{ii. Publicación de laudos}

Tanto la ICC, ICDR y HKIAC tienen provisiones para la publicación de laudos arbitrales. La regulación de la ICC establece como regla general que se publicarán los laudos dos años después de su notificación, salvo que alguna de las partes manifieste expresamente su oposición. Debemos notar que, de acuerdo con las Notas a las partes y al Tribunal Arbitral para la conducción del arbitraje, "en caso de un acuerdo de confidencialidad que cubra ciertos aspectos del arbitraje o del laudo, la publicación estará sujeta al consentimiento específico de las partes". Por su parte, HKIAC publicará laudos una vez que haya borrado cualquier dato que permita identificar a una de las partes. Finalmente, ICDR solamente publicará los laudos cuando exista consentimiento expreso entre las partes o la ley lo requiera.

73 Consulta realizada el día 18 de julio de 2020. Disponible en: https:// www.icdr.org/sites/default/files/document_repository/AAA241_ICDR_Time_and_C ost_Study.pdf?_ga=2.82242390.1562198379.1594043737-324050970.1554421611

74 Consulta realizada el día 18 de julio de 2020. Disponible en: https://www.hkiac.org/aboutus/statistics 
Nos encontramos frente a tres distintos estándares; sin embargo, en cualquier caso, la objeción de cualquiera de las partes, impedirá la publicación del laudo. Esta es una clara muestra de los balances entre confidencialidad y transparencia75.

La importancia de la publicación de laudos radica en que

"el potencial precedente de los laudos arbitrales constituye un bien público. Aunque los laudos no están sujetos a un sistema formal de stare decisis, las decisiones de los tribunales arbitrales pueden aportar una contribución sustancial e importante a la jurisprudencia comercial, orientando a los futuros tribunales y a los tribunales nacionales cuando se les planteen controversias similares76".

Como resaltar Born, aun cuando se considere que el laudo constituye un procedente, su publicación puede generar beneficios de eficiencia, coherencia y previsibilidad, ya que las decisiones previas pueden orientar a los tribunales posteriores77.

De los reglamentos peruanos revisados, tanto la CCL como Amcham contemplan la publicación de laudos comerciales78. Sin embargo, hasta donde alcanza nuestro conocimiento, solamente la CCL publica laudos comerciales con fines académicos, siempre que ninguna parte objete la publicación. Esta medida es positiva y también debería ser seguida por las otras instituciones debido a los beneficios que ya hemos mencionado.

Sin perjuicio de ello, la puesta en marcha de esta medida debe ser con bastante cautela en la medida que el número de casos comerciales es tan alto y podría "filtrarse" información que las partes pretendían que se mantengan confidenciales.

\section{iii. Ayuda en la selección de los árbitros y evaluación}

Una de las medidas recientes por parte de la ICC es la colaboración a las partes en la selección de miembros del tribunal arbitral. De acuerdo con las ya referidas Notas, las partes de manera conjunta o los árbitros al designar al presidente del Tribunal, podrán pedir asistencia de la Secretaría. Esta última propondrá candidatos o brindará información no confidencial sobre las personas propuestas. De igual manera, la Secretaría podrá también contactar a las personas propuestas como árbitros para informarse sobre su experiencia, disponibilidad y posibles conflictos de intereses.

75 Precisamente, en la nota de la Secretaría de UNCITRAL No A/CN9/207, se señaló que lo mejor es dejar la decisión a las partes.

76 COMRIE-THOMSON, Paul. “A Statement of Arbitral Jurisprudence: The Case for a National Law Obligation to Publish International Commercial Arbitral Awards". En Journal of International Arbitration, Kluwer Law International, vol. 34, núm. 2, 2017, p. 282

77 BORN, Gary. International Commercial Arbitration. Austin: Aspen publishers, 2da edición, 2014, p. 2822.

78 Recordemos que la publicación de laudos en donde interviene una parte estatal está regulada en la Ley. 
El ICDR tiene un sistema de lista para la selección de árbitros, en la que incluye las calificaciones solicitadas por las partes, la nacionalidad específica, especialización o experiencia, idioma y conocimiento del derecho sustantivo. El Centro elabora la lista con los aportes de las partes y emite una lista de nombre. Las partes pueden objetar a uno de la lista, sin expresar razones y deben numerar sus preferencias. Los árbitros con mayores calificaciones son elegidos, luego de verificar sus conflictos de interés79. En el caso de LCIA en el pasado ha aceptado varios tipos de procedimientos por lista (nombres dados por las partes o por el centro); su objetivo es facilitar el proceso de nombramientoso.

HKIAC ha lanzado un sistema para que las partes puedan evaluar el desempeño de los árbitros. Se trata de un esfuerzo para que las partes tengan mejor información para designar árbitros. La evaluación contempla los siguientes aspectos: i) Preparación general y familiaridad con las leyes aplicables y reglas; ii) Habilidad para facilitar la justicia, neutralidad y proceso efectivo; iii) Habilidades de manejo del caso; iv) Habilidad para el manejo de las pruebas; v) Habilidades de comunicación; y, vi) Proceso de decisións1.

Por su parte, Julio Olórtegui y José María de la Jara proponen una serie de preguntas a los árbitros, que deberán ser publicadas por los centros arbitrales sobre cinco asuntos: i) elección del tribunal; ii) disponibilidad de tiempo y método de trabajo; iii) estilo de decisiones; iv) pruebas y audiencias; y, v) deliberación y emisión del laudo82. Esta información también podría ser relevante para la asistencia a las partes en la selección de árbitros.

Ninguna de las instituciones de nuestro país ha adoptado medidas similares. La adopción de medidas destinadas a evaluar a los árbitros resulta más compleja de implementar, pues el mecanismo debe evitar los sesgos de la parte que perdió el arbitraje. En cambio, consideramos altamente factibles que las instituciones pueden a través de sus cortes, colaborar con la designación de árbitros a través de una lista, siempre que las partes lo hayan acordado previamente.

\section{iv. Publicación de información sobre las recusaciones}

De acuerdo con la Guía para las Partes del LCIA83, este centro de arbitraje publicará extractos o resúmenes de las decisiones de la Corte con respecto a la recusación de

79 MARTINEZ, Luis. “The ICDR's Arbitrator Appointment Process - The Institutional Role and Available Options". En American Bar Association, Section of International Law, vol. 1, 2013, p. 23

80 SCHERER, Maxi, RICHMAN, Lisa, et al. Arbitrating under the 2014 LCIA Rules: A User's Guide. Kluwer Law International, 2015, p. 109

81 Para mayor información ver: https://www.hkiac.org/news/rate-your-experience-hkiaclaunches-arbitration-evaluation-system

82 DE LA JARA, José María y OLÓRTEGUI, Julio. "No pain, no gain: Nueve propuestas para mejorar el futuro del arbitraje internacional". En Themis Revista de Derecho, núm. 71, 2017, p. 95.

83 Consulta realizada el día 18 de julio de 2020. Disponible en: https://www.lcia.org//adrservices/lcia-notes-for-parties.aspx 
los árbitros. Esta información es de crucial importancia tanto para las partes como para los propios árbitros, a fin de determinar cuál es el estándar.

Su importancia radica en que si bien no son un precedente,

"cada una de las decisiones de recusación ofrece una orientación útil en relación con la integridad del arbitraje y, en muchos casos, las decisiones abordan múltiples motivos de recusación. Varias de las decisiones se refieren a casos sencillos de recusaciones claramente meritorias o frívolas. No obstante, incluso esas decisiones son valiosas porque ayudan a enmarcar los límites de lo que puede considerarse una cuestión de buena fe de independencia o imparcialidad arbitral 84 ".

En el caso de HKIAC, en el 2014 emitió una nota con respecto a la recusación de los árbitros. En esta se señaló expresamente que la Corte no estaba obligada a motivar sus decisiones. Situación que, desde nuestra perspectiva, es contraria a los estándares de transparencia. En el caso de la ICC, la Corte puede comunicar las razones de su decisión sobre una recusación siempre que sea solicitado por una parte85.

De los reglamentos analizados en el Perú, solamente Amcham establece expresamente que su corte resuelve de manera motivada la recusación. Sin embargo, tanto la CCL como la PUCP motivan sus decisiones sobre recusaciones.

De la misma manera que se publican laudos arbitrales, consideramos que sería un acierto por parte de las instituciones locales publicar sus decisiones sobre recusaciones, siempre que sea posible anonimizar a las partes y sus datos de identificación86. De esta manera, se genera mayor predictibilidad y se comunica a los usuarios cuáles situaciones no son aceptables y dan lugar a una recusación.

En todos los casos mencionados, como bien observa el profesor Born, se intenta mantener el deber de confidencialidad que tanto interesa a las partes:

"Estos diversos acontecimientos han hecho que la comunidad y el proceso de arbitraje internacional sean claramente accesibles. No obstante, y lo que es más importante, no tienen en principio ningún efecto sobre la divulgación de detalles relativos a las presentaciones y pruebas en los arbitrajes en curso, las audiencias arbitrales o las deliberaciones de los árbitros. Estos aspectos del procedimiento arbitral han conservado su carácter presuntamente confidencial, precisamente porque son los que más directamente sirven a los objetivos básicos del proceso arbitral 87 ".

84 WALSH, Thomas y TEITELBAUM, Ruth. "The LCIA Court Decisions on Challenges to Arbitrators: An Introduction". En William W. Park (ed.), Arbitration International Special Edition on Arbitrator Challenges, Kluwer Law International, 2011, p. 284.

85 Numeral 14 de las Notas de la ICC.

86 La PUCP ha publicado la cantidad de recusaciones y renuncias ante su Corte; sin embargo, consideramos que podrían ir un paso más allá.

87 BORN, Gary. International Commercial Arbitration. Austin: Aspen publishers, 2da edición, 2014, p. 2822. 
En cuanto a los costos y beneficios de implementar estas medidas, comparto la posición de Rogers, quien señala que a pesar de no son fácilmente cuantificable ambos factores, un sistema transparente compensa sus costos88. Además, como ya hemos señalado generará mayor confianza en la institución por parte de los usuarios, lo que permitirá seguir expandiendo las fronteras del arbitraje.

\section{Conclusiones}

La confidencialidad se mantiene como uno de los elementos esenciales del arbitraje comercial en el mundo, desde la perspectiva de los usuarios. Desde nuestra perspectiva, la regla general que debe primar es el pacto de las partes, ya sea mediante un acuerdo expreso en el convenio arbitral que se pronuncie sobre la confidencialidad o la remisión a un reglamento arbitral o una jurisdicción que tenga una disposición expresa. Si se trata de una industria o una relación en la que la confidencialidad es un aspecto central (energía, construcción, tecnología, entre otros), se debe buscar claridad en cuanto al alcance tanto subjetivo como objetivo de este deber.

En contraposición, la transparencia es una tendencia actual que ha calado con profundidad en diversos usuarios del arbitraje. Principalmente, en el arbitraje en el que interviene el Estado y el arbitraje de Inversión existen amplias medidas de transparencia. Sin embargo, inclusive en estos casos resulta controvertido la publicidad de las actuaciones arbitrales durante el arbitraje, pues la opinión pública podría ejercer una indebida presión sobre los árbitros. Situación que se buscaba evitar al pactar arbitraje. Aunque controvertida, la reciente modificación del Estado peruano al menos se cuida en este aspecto y dispone la publicidad de las actuaciones arbitrales una vez concluido el procedimiento.

En cuanto al arbitraje comercial, desde nuestra perspectiva, el enfoque para su correcto funcionamiento con la confidencialidad consiste en que el objetivo de la transparencia, debe ser brindar un mayor flujo de información a los usuarios del arbitraje, respecto a los árbitros, su desempeño, los potenciales conflictos de interés, entre otros.

Las instituciones arbitrales son las llamadas a llevar a cabo las reformas necesarias, para compatibilizar los principios de transparencia y confidencialidad, con el objetivo de brindar mejor información a los usuarios del arbitraje. Este proceso, además, mejorará la toma decisiones de los usuarios con respecto a sus controversias.

Por lo tanto, la eficacia de las reformas destinadas a brindar mayor transparencia dependerá de que no se destruya uno de los elementos más valorados por las partes en el arbitraje comercial, la confidencialidad.

88 ROGERS, Catherine. Transparency in International Commercial Arbitration. Penn State Law eLibrary, 2006, p. 1327. 